\title{
Correction of anterior mitral prolapse: The parachute technique
}

\author{
Konstantinos Zannis, MD, ${ }^{\mathrm{a}}$ Laurens Mitchell-Heggs, MD, ${ }^{\mathrm{a}}$ Valentina Di Nitto, MD, ${ }^{\mathrm{a}}$ \\ Matthias E. W. Kirsch, MD, PhD, ${ }^{\mathrm{b}}$ Milena Noghin, MD, ${ }^{\mathrm{a}}$ Gabriel Ghorayeb, MD, ${ }^{\mathrm{a}}$ and Arrigo Lessana, $\mathrm{MD}^{\mathrm{a}}$
}

Objectives: To evaluate a new surgical technique for the correction of anterior mitral leaflet prolapse.

\begin{abstract}
Methods: From October 2006 to November 2011, 44 consecutive patients (28 males, mean age $55 \pm 13$ years) underwent mitral valve repair because of anterior mitral leaflet prolapse. Echocardiography was performed to evaluate the distance from the tip of each papillary muscle to the annular plane. A specially designed caliper was used to manufacture a parachute-like device, by looping a 4-0 polytetrafluoroethylene suture between a Dacron strip and Teflon felt pledget, according to the preoperative echocardiographic measurements. This parachute was then used to resuspend the anterior mitral leaflet to the corresponding papillary muscle. Of the 44 patients, $35(80 \%)$ required concomitant posterior leaflet repair. Additional procedures were required in 16 patients $(36 \%)$. The preoperative logistic European System for Cardiac Operative Risk Evaluation was $4.3 \pm 6.9$.
\end{abstract}

Results: The clinical and echocardiographic follow-up were complete. The total follow-up was 1031 patientmonths and averaged $23.4 \pm 17.2$ months per patient. The overall mortality rate was $4.5 \%(\mathrm{n}=2)$. Also, 2 patients $(4.5 \%)$ with recurrent mitral regurgitation required mitral valve replacement, 1 on the first postoperative day and 1 after 13 months. In the latter patient, histologic analysis showed complete endothelialization of the Dacron strip. At follow-up, all non-reoperated survivors $(\mathrm{n}=40)$ were in New York Heart Association class I, with no regurgitation in 40 patients $(93 \%)$ and grade $2+$ mitral regurgitation in $3(7 \%)$.

Conclusions: This technique offers a simple and reproducible solution for correction of anterior leaflet prolapse. Echocardiography can reliably evaluate the length of the chordae. However, the long-term results must be evaluated and compared with other surgical strategies. (J Thorac Cardiovasc Surg 2012;143:S24-8)

The benefits of mitral valve repair over valve replacement have made mitral valve repair the treatment of choice for patients with mitral regurgitation (MR). Although posterior leaflet repair is well codified, anterior leaflet repair can be challenging. The introduction of polytetrafluoroethylene (PTFE) sutures for chordal replacement have simplified this procedure. ${ }^{1}$ However, the length of the artificial chordae remains an issue. In the present report, we present an alternative method for anterior mitral leaflet (AML) valve repair and our short-term results.

\section{PATIENTS AND METHODS \\ Patients}

The local ethics committee approved the present study, and the need for patient consent was waived because of the retrospective nature of the study.

\footnotetext{
From the Department of Cardiac Pathology, ${ }^{a}$ Institut Mutualiste Montsouris, Paris, France; and Department of Cardiac Surgery, ${ }^{\mathrm{b}}$ Institut de Cardiologie, Hôpital Pitié-Salpêtrière, Groupe Hospitalier Pitié-Salpêtrière, Paris, France.

Disclosures: Konstantinos Zannis, Laurens Mitchell-Heggs, Valentina Di Nitto, Matthias E. W. Kirsch, Milena Noghin, Gabriel Ghorayeb, and Arrigo Lessana have nothing to disclose with regard to commercial support.

Presented at The American Association for Thoracic Surgery Mitral Conclave, New York, New York, May 5-6, 2011.

Received for publication May 15, 2011; revisions received Sept 22, 2011; accepted for publication Oct 20, 2011; available ahead of print Dec 6, 2011.

Address for reprints: Konstantinos Zannis, MD, L'Institut Mutualiste Montsouris, 42, Boulevard Jourdan, Paris 75674 Cedex 14, France (E-mail: konstantinos.zannis@ imm.fr).

0022-5223/ $\$ 36.00$

Copyright (C) 2012 by The American Association for Thoracic Surgery doi:10.1016/j.jtcvs.2011.10.034
}

From October 2006 to November 2011, 340 patients with MR underwent mitral valve repair at our institution. Of these, 44 consecutive patients $(13 \% ; 28$ males, mean age, $55 \pm 13$ years) presented with AML prolapse and underwent mitral valve repair using the parachute technique. These 44 patients formed the study cohort.

\section{Echocardiography}

Transthoracic preoperative echocardiography was performed in all patients and allowed measurement of the distance from the tip of each papillary muscle to the annular plane at end-systole in an apical view (Figure 1, A). The length of the free edge of the entire anterior mitral leaflet was also measured in the short-axis parasternal view at end-diastole. Perioperative transesophageal echocardiography was mandatory. When poor echogenicity impeded a correct evaluation of the preoperative measurements, perioperative transesophageal off-pump measurements were used as the proxy.

Postoperative transesophageal echocardiography was used to evaluate valve competence according to the presence of color Doppler residual regurgitation, the presence of a satisfactory coaptation height, and the absence of systolic anterior motion.

\section{Surgical Technique}

A parachute-like device ${ }^{2}$ was constructed by looping a 4-0 polytetrafluoroethylene (Gore-Tex, WL Gore \& Associates, Flagstaff, Arizona) suture between a 2.5-mm-wide Dacron strip (Bard Sauvage, Tempe, Arizona) and a Teflon felt pledget. A dedicated caliper (Delacroix-Chevalier, Paris, France) was used to ensure adequate length of the PTFE loops and of the Dacron strip according to the preoperative echocardiographic measurements. When necessary, a separate device was manufactured for each papillary muscle.

Median sternotomy or right thoracotomy was then performed and cardiopulmonary bypass was used in normothermia. Myocardial protection was obtained by intermittent antegrade and retrograde normothermic blood 


\section{Abbreviations and Acronyms \\ $\mathrm{AML}=$ anterior mitral leaflet \\ MR = mitral regurgitation \\ PTFE $=$ polytetrafluoroethylene}

cardioplegia. The mitral valve was approached either by the left atrium through Sondergard's groove or using a biatrial approach, depending on the concomitant procedures and surgeon preference.

\section{Valve Inspection}

A systematic inspection of the valve leaflets, mitral annulus, native chordae, and papillary muscle position was performed. The prolapsing segment with elongated or ruptured chordae of the AML was identified and stay sutures were placed on either side. The segment between these stay sutures was considered the region requiring chordal support. In all cases, the match between the preoperative echocardiographic findings and perioperative evaluation was good. No additional intraoperative measures were performed by the operating surgeon. Particular attention was paid to the papillary head to identify the future suture site of the neosubvalvular apparatus.

\section{Parachute Landing}

The parachute-like device was brought into the operating field, and the PTFE suture was inserted adjacent to the papillary muscle head, affixed to a pledget on each side. Next, the upper part of the "parachute" was sutured on the rough zone of the prolapsing anterior leaflet using a running 5-0 polypropylene suture (Figure 1,B).

\section{Postoperative Antithrombotic Treatment}

All patients received intravenous heparin until epicardial lead ablation, followed by oral anticoagulation using fluindione for the first 3 postoperative months. The international normalized ratio was targeted at 2.5 to 3.5 . Thereafter, anticoagulation was suspended in the absence of other indications.

\section{Data Collection}

The preoperative and operative data were recorded in a computerized data registry. A preoperative risk assessment was performed according to the definitions of the European System for Cardiac Operative Risk Evaluation (EuroSCORE). ${ }^{3}$

\section{Follow-up}

The patients were systematically followed up at 1 and 3 months and every 6 months thereafter. The clinical status and echocardiographic data were recorded and entered into our database. Postoperative events were compiled and analyzed according to the guidelines for reporting morbidity and mortality after cardiac valvular operations. ${ }^{4}$ Only the latest echocardiographic data were considered for the present study.

\section{Statistical Analysis}

Statistical analysis was performed using the Statistical Package for Social Sciences (SPSS, Chicago, Illinois). Continuous variables are expressed as the mean \pm standard deviation and were compared using paired 2-tailed Student's $t$ tests. Categorical variables are expressed as percentages and compared using the chi-square test. A 2-tailed probability value of less than .05 was taken to indicate statistical significance. The survival data were analyzed using standard Kaplan-Meier actuarial techniques for estimation of survival probabilities.

\section{RESULTS \\ Patients}

All 44 patients had moderate to severe MR due to AML prolapse. The preoperative risk factors, MI etiology, and New York Heart Association preoperative class are listed on Table 1. The preoperative logistic EuroSCORE was $4.3 \pm 6.9$.

Of the 44 patients, $35(80 \%)$ presented with bileaflet prolapse requiring concomitant posterior leaflet repair (Table 2).

\section{Procedure}

Mitral valve repair was feasible in all patients. Additional AML repair procedures were performed in 7 patients $(16 \%)$ and included Alfieri stitch in 1 patient $(2.3 \%)$ and anterior leaflet triangular resection in $6(13.6 \%)$. Mitral annuloplasty was performed in all patients using a complete ring as follows: a Carpentier Edwards Physio ring (Edwards Lifescience, Irvine, California) in 19 patients (43\%), a Medtronic Duran annuloplasty ring (Medtronic, Minneapolis, Minnesota) in 16 patients $(36 \%)$, and Sorin Memo 3-dimensional annuloplasty ring (Sorin Biomedica Cardio S.r.L., Saluggia, Italy) in 9 patients $(20 \%)$. The mean annular size was $35.3 \pm 2.9$. Other procedures were required in 16 patients $(36 \%)$, including tricuspid valve repair in 9 $(20 \%)$, atrial septal defect repair in $4(9 \%)$, atrial fibrillation ablation in $2(4.5 \%)$, and coronary artery bypass grafting in $1(2.3 \%)$. The mean cardiopulmonary bypass and aortic crossclamp time was $118 \pm 29$ and $94 \pm 25$ minutes, respectively. Intraoperative echocardiography detected residual grade $2+$ MR in 1 patient $(2.3 \%)$ that was corrected by an Alfieri stitch. Systolic anterior motion was noted in 1 patient $(2.3 \%)$ and was managed successfully with short-acting $\beta$-blockers and volume expansion. Postoperative complications included re-exploration for bleeding in 2 patients $(4.5 \%)$ and transient motor deficit in 2 patients $(4.5 \%)$.

\section{Mortality}

The overall mortality rate was $4.5 \%(n=2)$. One of the redo patients with previous Bentall surgery never emerged from the anesthesia, and postoperative cerebral imaging revealed diffuse cerebral injury. Another patient died late after surgery (160 days postoperatively) of progressive heart failure with recurrent valve insufficiency; the patient was also affected by uncontrolled leukemia.

\section{Reoperation}

Two patients $(4.5 \%)$ required mitral valve reoperations, both for recurrent MR. One patient required early reoperation on postoperative day 1 for parachute disinsertion resulting from loosening of the running 5-0 polypropylene suture. In this context, the operating surgeon opted for a straight replacement using a bioprosthesis. A second patient 

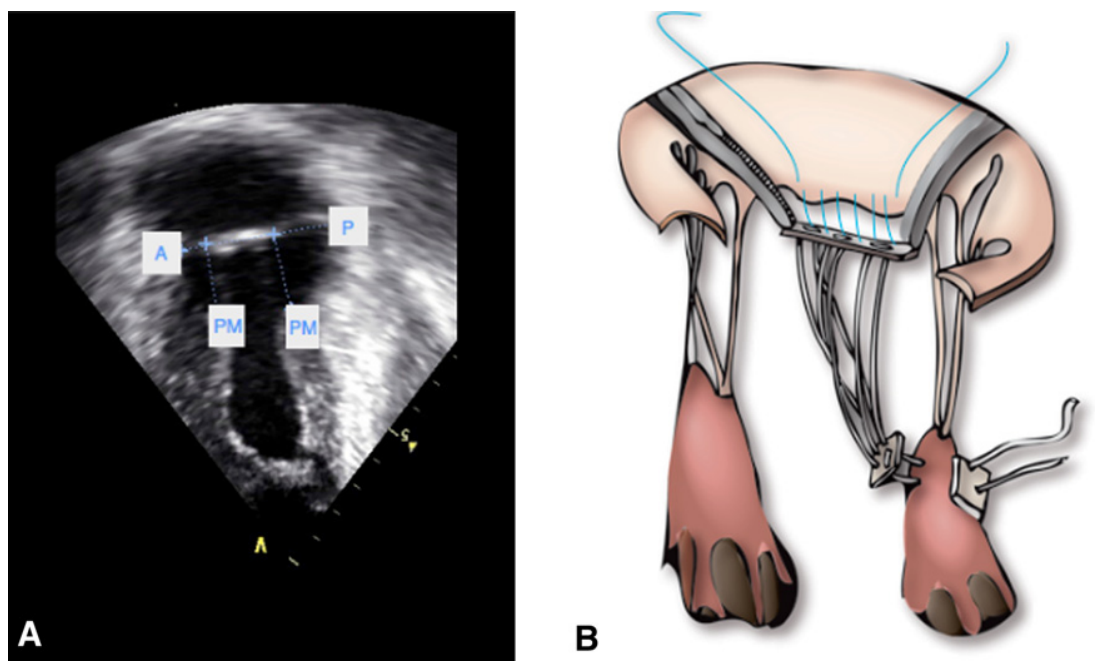

FIGURE 1. A, Preoperative echocardiogram measurements. A-P line, Annular plane; $P M$, papillary muscle. B, Parachute insertion.

required reoperation 1 year after the initial procedure. The intraoperative evaluation demonstrated that MR was related to dehiscence of the Alfieri stitch at the level of the posterior leaflet. Histologic analysis of the explanted valve showed complete endothelialization of the Dacron strip with no calcifications or stiffening.

\section{TABLE 1. Clinical data}

\begin{tabular}{lc}
\hline \multicolumn{1}{c}{ Variable } & Result \\
\hline Patient demographics & \\
Mean age (y) & $55 \pm 13$ \\
Male gender (n) & $28(64)$ \\
Risk factors (n) & \\
Ejection fraction $<50 \%$ & $3(7)$ \\
COPD & $2(5)$ \\
Neurologic history & $1(2)$ \\
Previous cardiac surgery & $3(7)$ \\
Serum creatinine $>200 \mu$ mol/L & $0(0)$ \\
Active endocarditis & $2(5)$ \\
Critical preoperative state & $0(0)$ \\
Acute myocardial infarction & $0(0)$ \\
Pulmonary hypertension $>60$ mm Hg & $3(7)$ \\
Emergency & $0(0)$ \\
Peripheral vascular disease & $2(5)$ \\
Additive EuroSCORE & $4.1 \pm 2.3$ \\
Logistic EuroSCORE & $4.3 \pm 6.9$ \\
Etiology of MI (n) & \\
Degenerative & $42(91)$ \\
Endocarditis & $1(2)$ \\
Ischemic papillary muscle rupture & $1(2)$ \\
NYHA functional class (n) & \\
I & $15(34)$ \\
II & $26(59)$ \\
III & $1(2)$ \\
IV & $2(5)$ \\
\hline Dan par & \\
\hline &
\end{tabular}

Data in parentheses are percentages. $C O P D$, Chronic obstructive pulmonary disease; EuroSCORE, European System for Cardiac Operative Risk Evaluation; MI, myocardial infarction; NYHA, New York Heart Association.
No patient developed mitral valve endocarditis during follow-up.

\section{Embolic and Bleeding Events}

One patient $(2.3 \%)$ experienced transient ischemic attack (diplopia) on postoperative day 34 . No patient presented with bleeding complications.

\section{Clinical and Echocardiographic Follow-up}

Clinical follow-up was complete and averaged $23.4 \pm$ 17.2 months (range, 1 to 51). The total follow-up was 1031 patient-months and averaged $23.4 \pm 17.2$ months per patient. At the latest follow-up examination, all nonreoperated patients $(n=40)$ were in New York Heart Association class I.

Echocardiographic follow-up was complete and averaged $17.4 \pm 14.9$ months, with a total follow-up of 735.8 patient-months. Of the 44 patients, 29 (66\%) had an echocardiographic follow-up of at least 6 months. Thirty-seven patients $(93 \%)$ had no or trivial MR. Three patients $(7 \%)$ had mild MR (grade 2 or less). The ventricular and atrial

TABLE 2. Procedures

\begin{tabular}{lc}
\hline \multicolumn{1}{c}{ Procedure } & Patients (n) \\
\hline Parachute to AML and annuloplasty & $44(100)$ \\
Parachute from APM to A1-A2 and from PPM to A2-A3 & $30(68)$ \\
Parachute from the PPM to A2-A3 & $10(23)$ \\
Parachute from the APM to A1-A2 & $4(9)$ \\
Associated posterior leaflet prolapse repair & $35(80)$ \\
Resection and folding & $30(68)$ \\
Resection and plication & $4(10)$ \\
Parachute to posterior leaflet & $1(2)$ \\
Associated procedures* & $16(39)$ \\
\hline
\end{tabular}

Data in parentheses are percentages. $A P M$, Anterior papillary muscle; $P P M$, posterior papillary muscle. *Tricuspid valve repair in 9 , atrial septal defect repair in 4 , atrial fibrillation ablation in 2, coronary artery bypass grafting in 1 . 
TABLE 3. Cardiac chamber dimensions

\begin{tabular}{lccc}
\hline Variable & Preoperative & Postoperative & $\boldsymbol{P}$ value \\
\hline LVESD & $36.0 \pm 9.4$ & $35.9 \pm 10.0$ & .98 \\
LVDD & $59.5 \pm 11.0$ & $55.5 \pm 13.9$ & .012 \\
LAD & $49.0 \pm 13.6$ & $39.3 \pm 12.9$ & .02 \\
\hline
\end{tabular}

$L V E S D$, Left ventricular end systolic diameter; $L V D D$, left ventricular diastolic diameter; $L A D$, left atrial diameter.

dimensions are described at Table 3 and compared with the preoperative measurements.

\section{DISCUSSION}

Anterior leaflet prolapse, although less common than posterior prolapse, affects up to one third of patients with MR. ${ }^{5}$ In the early days of conservative mitral valve surgery, treatment of anterior leaflet prolapse was considered a relative contraindication to mitral valve repair. Carpentier's technique ${ }^{6}$ of chordae transfer from the posterior to the anterior leaflet then introduced a standardized method for treating AML prolapse. Extending this concept, Lessana and colleagues ${ }^{7,8}$ proposed transposing a large bundle of the posterior leaflet with its multiple chordal attachments for the treatment of large AML prolapsing areas. However, these techniques presumed that the posterior leaflet is exempt from the pathologic process. This was not the case in the present patient series, in which only $20 \%$ of the patients had an isolated AML prolapse.

The first large cohort of patients with mitral prolapse treated with PTFE artificial chordae was described by David and colleagues. ${ }^{1}$ Since then, PTFE sutures have increased in popularity and have demonstrated good and stable results. The use of neochordae offers the theoretical advantage of obtaining larger coaptation surfaces, owing to the absence of tissue resection. However, the neochordal length and the correct number of neochordal elements needed remains an issue.

Oppell and $\mathrm{Mohr}^{9}$ and others have described a simple method of determining the neochordal length, in which a caliper is used intraoperatively to measure the distance between the papillary muscle tip and the annular plane. The same caliper is used for the construction of 1 to 3 loops to resuspend the AML. Although this method is well described, it presumes that the pathologic process does not likewise affect the adjacent native chordae. Furthermore, the chordae are examined in a resting empty heart.

Since the early days of conservative mitral surgery, ${ }^{6}$ surgeons have learned the key to mitral competence is to keep the coaptation rim at the level of the annular plane. Using this principle, Calafiore ${ }^{10}$ described a method that combines preoperative echocardiographic data and intraoperative measures to determine the correct neochordae length.

However, these techniques use intraoperative measures on a resting heart. We hypothesized that basing our reconstruction strategy on a dynamic mitral valve analysis would be more physiologic. The distance between the papillary muscle head and mitral annular plane and the width of the prolapsing area were measured during preoperative bidimensional transthoracic echocardiography. The first measure was used to decide the length of the neochordal loops and the latter on whether to use 1 or 2 parachutes. When the prolapsing segment was too large, a second parachute was used to anchor the prolapsing AML to both papillary muscles to equilibrate the forces. We believe the parachute technique is an evolution of the original "flip-over" technique, ${ }^{7,8}$ in which the bundle of the posterior leaflet with its multiple chordal attachments was replaced by the Dacron strip and the neochordae, making repair possible even when posterior leaflet is prolapsing. ${ }^{2}$ In contrast to the technique of Von Oppell and Mohr, ${ }^{9}$ we constructed our loops with the interposition of a thin Dacron strip to reinforce the neochordae. Thus, the tension of mitral closure is not supported by a single stitch per loop but distributed over the Dacron strip surface by a running suture.

In the beginning of our experience, very large AML prolapses were addressed by triangular resection of some the excess tissue before parachute implantation. In the perennial concern of simplification and standardization of surgical techniques, we have completely abandoned AML triangular resection, and the running suture was used to crimp excessive tissue on the Dacron strip of the parachute.

We had some concern about the potential interactions between the Dacron strip and the native AML, with fibrosis and retraction possible that could affect the long-term results. In this patient cohort, we had to reoperate on 1 patient for late failure. Radiographic examination was performed under mammography conditions on the explanted AML (Faxitron, Wheeling, Illinois), and no calcifications were detected. Samples consisting of sections from the mitral annulus to the free edge of the AML were prepared and embedded in paraffin. Histopathologic study showed that the Dacron strip was embedded in a fibrous tissue and lined by endotheliform cells, confirming that the Dacron strip did not cause retraction or calcification. Also, the endotheliform cell lining suggests a low thromboembolic potential of the implanted material. In this cohort, most $(80 \%)$ of the patients had bileaflet prolapse due to mitral valve dystrophy. These patients with tissue excess and a high posterior leaflet were at risk of systolic anterior leaflet movement, which could be a concern. For these patients, our preferred treatment of posterior leaflet prolapse is quadrangular resection and folding, allowing one to lower the height of the posterior leaflet and to bring the coaptation zone toward the posterior part of the annulus. Systolic anterior leaflet movement was observed in 1 patient, but was reverted by intravascular volume expansion and $\beta$-blockers. During the study period, 2-dimensional transthoracic echocardiography was used to 
guide our reconstruction strategy. This technique allowed a full, comprehensive anatomic study of the mitral valve with good localization of the prolapse. The advent of 3-dimensional echocardiography could be of interest in the near future for even more precise valve analysis and better communication between the echocardiographists and surgeons.

\section{CONCLUSIONS}

The parachute technique is feasible and offers an attractive alternative for patients with large anterior leaflet prolapse as determined by a comprehensive preoperative echocardiographic evaluation. However, the long-term results need to be evaluated and compared with other surgical strategies.

\section{References}

1. David TE, Omran A, Armstrong S, Sun Z, Ivanov J. Long-term results of mitral valve repair for myxomatous disease with and without chordal replacement with expanded polytetrafluoroethylene sutures. J Thorac Cardiovasc Surg. 1998;115: 1279-85. 1276.
2. Scorsin M, Al-Attar N, Lessana A. A novel technique of utilizing artificial chordae for repair of mitral valve prolapse. J Thorac Cardiovasc Surg. 2007;134:1072-3.

3. Nashef SA, Roques F, Michel P, Gauducheau E, Lemeshow S, Salamon R. European System for Cardiac Operative Risk Evaluation (EuroSCORE). Eur J Cardiothorac Surg. 1999;16:9-13.

4. Akins CW, Miller DC, Turina MI, Kouchoukos NT, Blackstone EH, Grunkemeier GL, et al. Guidelines for reporting mortality and morbidity after cardiac valve interventions. J Thorac Cardiovasc Surg. 2008;135:732-8.

5. Grossi EA, Galloway AC, LeBoutillier M III, Steinberg B, Baumann FG, Delianides J, et al. Anterior leaflet procedures during mitral valve repair do not adversely influence long-term outcome. J Am Coll Cardiol. 1995;25:134-6.

6. Carpentier A. Cardiac valve surgery: the "French correction." J Thorac Cardiovasc Surg. 1983;86:323-37.

7. Lessana A, Escorsin M, Romano M, Ades F, Vergoni W, Lorenzoni D, Menozzi C, Monducci I. Transposition of posterior leaflet for treatment of ruptured main chordae of the anterior mitral leaflet. J Thorac Cardiovasc Surg. 1985;89:804-6

8. Lessana A, Romano M, Lutfalla G, Carbone C, Palsky E, Amalou SA, Escorsin M. Treatment of ruptured or elongated anterior mitral valve chordae by partial transposition of the posterior leaflet: experience with 29 patients. Ann Thorac Surg. 1988;45:404-8.

9. von Oppell UO, Mohr FW. Chordal replacement for both minimally invasive and conventional mitral valve surgery using premeasured Gore-Tex loops. Ann Thorac Surg. 2000;70:2166-8.

10. Calafiore AM. Choice of artificial chordae length according to echocardiographic criteria. Ann Thorac Surg. 2006;81:375-7. 